\title{
On Profit Density Based Greedy Algorithm for a Resource Allocation Problem in Web Services
}

\author{
John Sum ${ }^{1,3}$, Jie $\mathrm{Wu}^{2}$ and Chi-sing Leung ${ }^{3}$ \\ ${ }^{1}$ Department of Information Management \\ Chung Shan Medical University \\ Taichung, Taiwan, ROC \\ ${ }^{2}$ Department of Computer Science and Engineering \\ Florida Atlantic University \\ Boca Raton, Florida 33431, USA \\ ${ }^{3}$ Department of Electronic Engineering \\ City University of Hong Kong \\ Kowloon Tong, KLN, Hong Kong
}




\begin{abstract}
Allocating limited computational resources to different clients is always a challenging problem to a web service provider (WSP). Profit density based greedy knapsack algorithm is one simple approach that can ensure near-optimal profit. However, profit gain is sometimes not the only factor concerned in making important management decisions. Other factors, such as the number of clients that a WSP can serve and the number of un-used resources that remain, are also important. By assuming that (a) the pricing curves of the buyer are all identical and their marginal utility (i.e. $\Delta$ Price $/ \Delta$ Size) is decreasing, (b) the resource is divisible, (c) the resource quantity each client requests follows uniform distribution $U[0,1]$; and (d) the available resource is constrained by $\bar{k}$; equations for the expected number of clients who can get the resource, denoted by $\langle b\rangle$, and the expected quantity of resource being allocated, denoted by $\langle s\rangle$, are derived analytically. By observing the numerical plots of $\langle b\rangle$ and $\langle s\rangle$ against the number of clients $n$, it is found that $\langle b\rangle \approx n$ for $n \leq 2 \bar{k}$ and $\langle b\rangle \approx(-1+\sqrt{1+8 n \bar{k}}) / 2$ for $n \geq 2 \bar{k}$. Comparing with another simple selling mechanism, we call it first-come-first-serve (FCFS), it is found that resource allocation via greedy algorithm might not always be the best choice as far as the number of units being sold and the number of clients being served are concerned.
\end{abstract}

Keywords: knapsack problem, order statistics, profit density greedy algorithm, sum of random variables, uniform distribution 


\section{Introduction}

Extended from the ideas of software reuse and component based development, web service is a new paradigm and possibly a new direction for system development. A web services provider (WSP) makes application components available on the web. System developers can thus integrate those components (URLs) together to develop an application system. Certainly, the usage of these remote resources is usually not free. Allocating limited computational resources to clients in order to maximize the profit is one, but not the only, issue that every WSP needs to consider.

To solve this problem, one can apply an off-line allocation method. Let us consider a simple but normally not quite realistic situation. For clarification, Table 1 summarizes the notations appearing in the paper. Consider a WSP that has 20 servers available to support the service and 8 clients are willing to pay for their services.

\begin{tabular}{l|cccccccc}
\hline Client $i$ & B1 & B2 & B3 & B4 & B5 & B6 & B7 & B8 \\
\hline Server $k_{i}$ & 2 & 4 & 5 & 1 & 3 & 4 & 2 & 5 \\
Price $p_{i}$ & 10 & 30 & 35 & 6 & 15 & 18 & 12 & 35 \\
\hline
\end{tabular}

As resource is limited, the WSP has to select the most profitable clients and sign the service contracts. Obviously, this problem is essentially the $0 / 1$ knapsack problem $[4,5]$ (or recently it is called multi-units combinatorial auction problem [3]) that can be formulated by the following constraint optimization problem :

$$
\begin{array}{ll}
\text { Maximize } & 10 s_{1}+30 s_{2}+35 s_{3}+6 s_{4}+15 s_{5}+18 s_{6}+12 s_{7}+35 s_{8} \\
\text { Subject to } & 2 s_{1}+4 s_{2}+5 s_{3}+s_{4}+3 s_{5}+4 s_{6}+2 s_{7}+5 s_{8} \leq 20 \\
& s_{i} \in\{0,1\} \forall i=1, \ldots, 8 .
\end{array}
$$

For this simple problem, the WSP can profit 133 by allocating all 20 servers to B2, B3, B4, B5, B7 and B8. However, it is known that solving this constraint optimization problem will be intractable if the number of clients is large. Profit density based greedy algorithm is a nearoptimal alternative [5], which profits 128, by allocating 19 servers to B1, B2, B3, B4, B7 and B8. One server remains in the stock. 


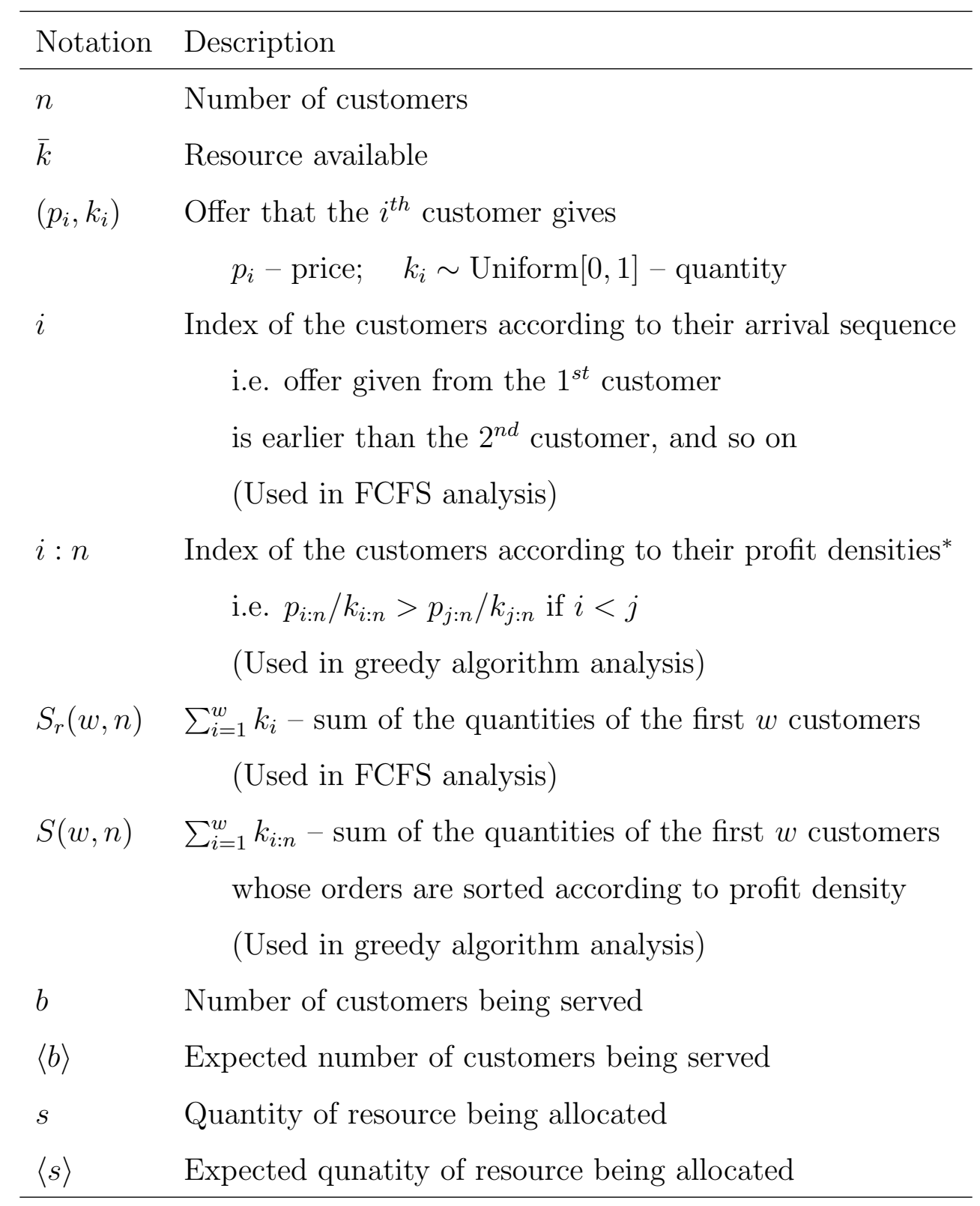

* Suppose there are 3 customers, their offers are $(3,0.2),(7,0.8)$, and $(5,0.4) .\left(p_{i}, k_{i}\right)$ for $i=1,2,3$ will be $(3,0.2),(7,0.8)$ and $(5,0.4)$ respectively. $\left(p_{i: 3}, k_{i: 3}\right)$ for $i=1,2,3$ will be $(3,0.2),(5,0.4)$ and $(7,0.8)$. Then $S_{r}(2,3)=1.0$ and $S(2,3)=0.6$.

Table 1: Notations. Here $k_{i} \sim$ Uniform $[0,1]$ means $k_{i}$ is random variable following uniform distribution. 
To trade-off the computational complexity, another even simpler mechanism called firstcome-first-serve (FCFS) - allocating resource to the client whenever the number of servers is available - can be used instead. In terms of profit gain, it is clear that profit density greedy algorithm is a better allocation method as it will ensure near optimal profit for number of customers is large. However, profit gain is sometimes not the only measure a company would like to know. Other factors, such as the number of clients it serves and the number of un-used resources remaining, are also important for making management decision.

In this paper, we assume that $\bar{k} \gg 1$ units of resource are available. Let the expected number of clients who can get the resource be $\langle b\rangle$, and the expected quantity of resource being allocated be $\langle s\rangle$, the purpose of the paper is to find out their relationships in terms of $n$ and $\bar{k}$. The essential technique being used is a formula derived by H. Weisberg [6] for a linear combination of order statistics and a formula derived by W. Feller (p.27 of [1]) for sum of uniformly random variables. The next section will describe the basic assumptions on $p_{i}$ and $k_{i}$. The profit denisty greedy algorithm and the FCFS mechanism will be presented. The expected number of customers $\langle b\rangle$ and the expected number of product being sold $\langle s\rangle$ for the mechanisms will be derived in Section 3. A discussion comparing greedy algorithm against FCFS method will be presented in Section 4. Then the conclusion will be presented in Section 5 .

\section{Greedy algorithm and FCFS}

Without loss of generality, we assume that $k_{i}$ is a random variable from $U(0,1)$. Next, we assume that the pricing function is marginal utility decreasing [2]. That is to say, a client would like to have a larger discount for a larger purchase. Mathematically, (i) $p^{\prime}\left(k_{i}\right) \geq p^{\prime}\left(k_{j}\right) \quad \forall 0 \leq k_{i} \leq k_{j} \leq$ 1 and (ii) $p(0) \geq 0$ and $p^{\prime}(0)>1$, where $p^{\prime}\left(k_{i}\right)$ is the first derivative of the function $p(k)$ at $k_{i}$. Two examplar functions satisfying the assumption are $p(k)=\alpha k+\beta$ and $p(k)=\alpha \log (1+k)+\beta$, where $k \in[0,1], \alpha$ and $\beta$ are non-negative constant values. It should be noted that the pricing function is a deterministic function depending solely on the quantity of resource requested. The following lemma will be used for later analysis. 
Lemma 1 For any non-negative real-valued function $f(x)$ that satisfies $f^{\prime}(x) \geq f^{\prime}(y) \geq 0$ for all $0 \leq x \leq y$ and $f(0) \geq 0$, then the following conditions hold : $(i) \frac{f(x)}{x} \geq f^{\prime}(x) ; \quad(i i) \quad \frac{f(x)}{x} \geq$ $\frac{f(y)}{y}$, for all $0 \leq x \leq y$.

Proof: The proof of the first inequality is straightforward. Since $f(x)=f(0)+\int_{0}^{x} f^{\prime}(u) d u \geq$ $f(0)+x f^{\prime}(x), f(x) / x \geq f^{\prime}(x)$. Using the fact that $f(y)=f(x)+\int_{x}^{y} f^{\prime}(u) d u$, then dividing both sides by $y$ and the condition, $f^{\prime}(x) \geq f^{\prime}(y)$, the following inequality can be obtained :

$$
\frac{f(y)}{y} \leq \frac{f(x)}{x}+\frac{(y-x)}{y}\left[f^{\prime}(x)-\frac{f(x)}{x}\right] .
$$

Since $\frac{f(x)}{x} \geq f^{\prime}(x)$, for all $y \geq x \geq 0, \frac{f(x)}{x} \geq \frac{f(y)}{y}$ and the proof is completed. Q.E.D.

\subsection{Greedy algorithm}

Suppose there are $n$ clients whose prices and quantities are $p_{1}, \ldots, p_{n}$ and $k_{1}, \ldots, k_{n}$ respectively. We call $\left(p_{i}, k_{i}\right)$ for all $i=1,2, \cdots, n$ the offers the clients give. Once all the offers have been collected, the WSP can apply the algorithm below to determine the allocation.

1: WAITFOR $\left(p_{i}, k_{i}\right), i=1, \ldots, n$;

2: $\operatorname{SORT}\left\{\frac{p_{i}}{k_{i}}\right\}$ s.t. $\frac{p_{i: n}}{k_{i: n}} \geq \frac{p_{j: n}}{k_{j: n}} \quad \forall i \leq j$;

3: $\operatorname{SET} C=\bar{k}$;

4: $\operatorname{SET} P=0$;

5: $\operatorname{SET} j=1$;

6: $\operatorname{WHILE}\left(C-k_{j: n}>0\right.$ and $\left.j \leq n\right)$

$$
\begin{aligned}
& C=C-k_{j: n} ; \\
& P=P+p_{j: n} ; \\
& j=j+1 ;
\end{aligned}
$$

END

First, their offers are ranked in descending order with respect to their profit density, i.e.

$$
\frac{p_{1: n}}{k_{1: n}} \geq \frac{p_{2: n}}{k_{2: n}} \geq \ldots \geq \frac{p_{n: n}}{k_{n: n}} .
$$


Then, we allocate the resource to the first $1: n, 2: n, \cdots, b: n$ clients, such that

$$
\sum_{i=1}^{b} k_{i: n} \leq \bar{k} ; \quad \sum_{i=1}^{b+1} k_{i: n}>\bar{k} .
$$

In accordance with the condition Equation (1), the condition $\frac{p_{i: n}}{k_{i: n}} \geq \frac{p_{j: n}}{k_{j: n}}$ implies that $k_{1: n} \leq$ $k_{2: n} \leq \ldots \leq k_{n: n}$ whenever price $p$ is a function of $k$ and its margainal utility is decreasing. So, $\frac{p_{1: n}}{k_{1: n}} \geq \frac{p_{2: n}}{k_{2: n}} \geq \ldots \geq \frac{p_{n: n}}{k_{n: n}}$ implies that $k_{1: n} \leq k_{2: n} \leq \ldots \leq k_{n: n}$ and their offers can be ranked in accordance with $k_{i}$. Again, the $\bar{k}$ units are allocated to the first $b$ bidders according to conditions in Equation (2).

\section{$2.2 \quad$ First-come-first-serve}

FCFS method is similar to selling products in a flea market. Once a customer has walked in and given an offer, the seller will check with the stock. The customer gets the product as long as there is available stock. One advantage of this FCFS method apart from its simplicity is that the customers do not have to wait. Besides, the seller has no need to anticipate the number $n$.

In web service provision, the WSP simply denies the service request whenever the available resource is not large enough to support the service. The FCFS method can be described by the following algorithm.

$$
\begin{aligned}
& \text { 1: } \operatorname{SET} C=\bar{k} ; \\
& \text { 2: } \operatorname{SET} P=0 ; \\
& \text { 3: } \operatorname{SET} j=1 ; \\
& \text { 4: } \operatorname{WHILE}\left(C-k_{j}>0 \text { and } j \leq n\right) \\
& \quad C=C-k_{j} ; \\
& \quad P=P+p_{j} ; \\
& \quad j=j+1 ; \\
& \quad \mathrm{END}
\end{aligned}
$$

Here, the index $i=1,2, \cdots$ represent the sequence of the offers made by the clients. In other words, the index $i=1,2,3, \cdots$ indicates their timing of visit. The $i^{\text {th }}$ client makes an offer 
earlier than the $j^{\text {th }}$ client if $i<j$. In this method, the WSP has no need to wait until all the offers have been collected. The decision is simply made by investigating the number of resources remaining. The resource is allocated to the first $1,2, \cdots, b$ clients

$$
\sum_{i=1}^{b} k_{i} \leq \bar{k} ; \quad \sum_{i=1}^{b+1} k_{i}>\bar{k}
$$

If the resource remaining is larger than the quantity requested by the walk-in client, the resource will be allocated accordingly.

\section{Analysis}

For the sake of analysis, let $S(w, n)=\sum_{i=1}^{w} k_{i: n}$ be the sum of units being sold to the $\{1$ : $n\},\{2: n\}, \cdots,\{b: n\}$ customers based on the profit density greedy algorithm. Similarly, we let $S_{r}(w, n)=\sum_{i=1}^{w} k_{i}$ be the sum of units being sold to the $1,2, \cdots, b$ customers based on the FCFS method.

\subsection{Greedy algorithm}

Since $k_{i}$ is a random variable drawn from uniform distribution for all $i=1,2, \cdots, n, k_{i: n}$ (after being sorted by profit density) is also a random variable drawn from uniform distribution for all $i=1,2, \cdots, n$.

$$
\begin{aligned}
S(w, n) & =\sum_{i=1}^{n} d_{i} k_{i: n} \\
d_{i} & = \begin{cases}1 & \forall i=1, \ldots, w \\
0 & \forall i=w+1, \ldots, n .\end{cases}
\end{aligned}
$$

The cumulative probability distribution $\operatorname{Pr}\{S(w, n) \leq \bar{k}\}$ can be evaluated by a formula derived by H. Weisberg in [6] (see Appendix),

$$
\begin{aligned}
\operatorname{Pr}\{S(w, n) \leq \bar{k}\} & =1-\sum_{j=1}^{r} \frac{\left(c_{j}-\bar{k}\right)^{n}}{c_{j} \prod_{j \neq i}\left(c_{j}-c_{i}\right)} \\
c_{i} & = \begin{cases}w-i+1 & \forall i=1, \ldots, w \\
0 & \forall i=w+1, \ldots, n .\end{cases}
\end{aligned}
$$


Unfortunately, this formula (as well as another formula from W. Feller [1]) cannot be reduced to a simple close form. To obtain the solution, one needs to do it numerically.

For the case that exactly $w$ customers are allocated with resources, it is equivalent to the case

$$
\{S(w, n) \leq \bar{k} \text { and } S(w+1, n)>\bar{k}\}
$$

Consider the following events,

$$
\begin{aligned}
& E_{1}=\{S(w, n) \leq \bar{k} \text { and } S(w+1, n) \leq \bar{k}\} \\
& E_{2}=\{S(w, n) \leq \bar{k} \text { and } S(w+1, n)>\bar{k}\} \\
& E_{3}=\{S(w, n)>\bar{k} \text { and } S(w+1, n) \leq \bar{k}\} \\
& E_{4}=\{S(w, n)>\bar{k} \text { and } S(w+1, n)>\bar{k}\}
\end{aligned}
$$

and the facts that (i) $\operatorname{Pr}\left\{E_{1}\right\}+\operatorname{Pr}\left\{E_{2}\right\}+\operatorname{Pr}\left\{E_{3}\right\}+\operatorname{Pr}\left\{E_{4}\right\}=1$ and (ii) $E_{3}=\phi$ the empty set, the probabilities for the events can readily be determined as follows :

$$
\begin{aligned}
& \operatorname{Pr}\left\{E_{1}\right\}=\operatorname{Pr}\{S(w+1, n) \leq \bar{k}\} \\
& \operatorname{Pr}\left\{E_{2}\right\}=\operatorname{Pr}\{S(w, n) \leq \bar{k}\}-\operatorname{Pr}\{S(w+1, n) \leq \bar{k}\} \\
& \operatorname{Pr}\left\{E_{3}\right\}=0 \\
& \operatorname{Pr}\left\{E_{4}\right\}=1-\operatorname{Pr}\{S(w, n) \leq \bar{k}\} .
\end{aligned}
$$

\subsubsection{Number of clients $\langle b\rangle$ being allocated with resources}

The probability of exactly $w$ clients being allocated with resources can be determined as follows :

$$
\operatorname{Pr}\{w \text { clients }\}= \begin{cases}\operatorname{Pr}\{S(w, n) \leq \bar{k}\}-\operatorname{Pr}\{S(w+1, n) \leq \bar{k}\} & \text { if } w<n \\ \operatorname{Pr}\{S(n, n) \leq \bar{k}\} & \text { if } w=n .\end{cases}
$$

This equation applies for all $w \geq \bar{k}$ and the evaluation of the $\operatorname{Pr}\{S(w, n) \leq \bar{k}\}$ can be based on Equation (6). Thus, the expected number of clients being allocated with resources, $\langle b\rangle$ can be determined by the following formula.

$$
\langle b\rangle=\sum_{w=1}^{n-1} w(\operatorname{Pr}\{S(w, n) \leq \bar{k}\}-\operatorname{Pr}\{S(w+1, n) \leq \bar{k}\})+n \operatorname{Pr}\{S(n, n) \leq \bar{k}\} .
$$




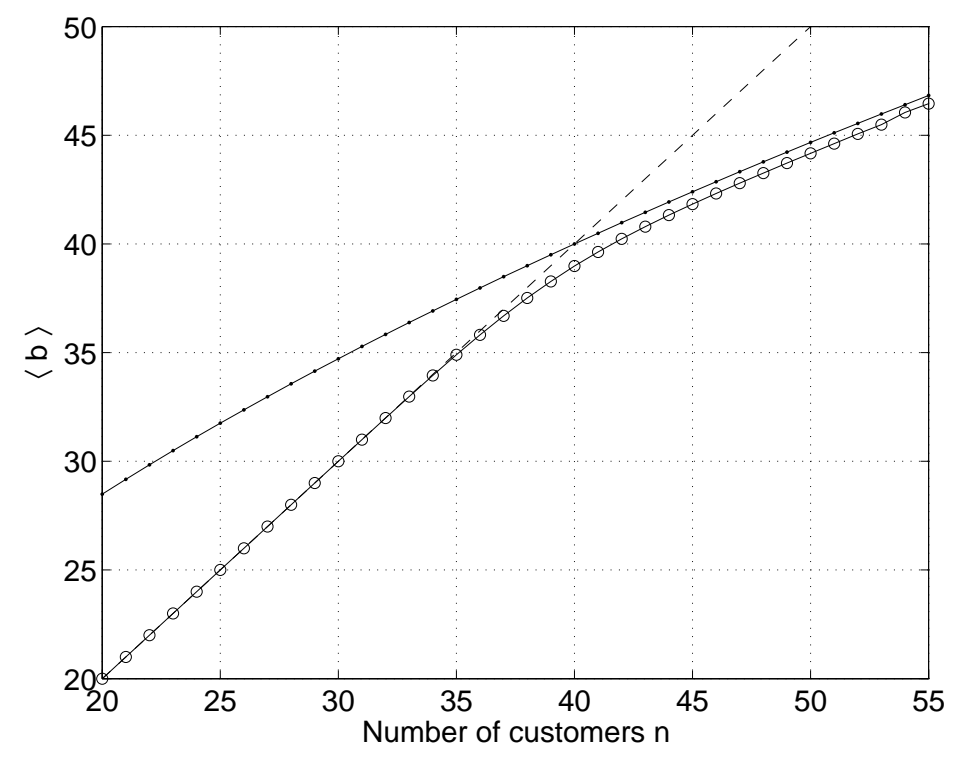

Figure 1: The expected number of clients being allocated with resource against the number of customers for $\bar{k}=20$ is shown by solid line with circles. The dotted line corresponds to $\langle b\rangle=n$ and the dot-solid line corresponds to $\langle b\rangle=\frac{-1+\sqrt{1+8 n \bar{k}}}{2}$.

Since $\operatorname{Pr}\{b=w\}=1$ for all $w \leq \bar{k}$, the summation can be started with $w=\bar{k}$.

$$
\langle b\rangle=\sum_{w=\bar{k}}^{n-1} w(\operatorname{Pr}\{S(w, n) \leq \bar{k}\}-\operatorname{Pr}\{S(w+1, n) \leq \bar{k}\})+n \operatorname{Pr}\{S(n, n) \leq \bar{k}\} .
$$

It is a function dependant on $n$ and $\bar{k}$. Once $n$ and $\bar{k}$ are known, $\langle b\rangle$ can be evaluated numerically. Figure 1 illustrates the case when $\bar{k}=20$. We have also plotted the curve for the cases when $\bar{k}$ equals to 30 and 40 respectively. All of them show the same shape. It can be observed that for $n \leq 2 \bar{k}$ and $n \geq 2 \bar{k},\langle b\rangle$ can be approximated as follows :

$$
\langle b\rangle \approx \begin{cases}n & \text { if } n \leq 2 \bar{k} \\ \frac{-1+\sqrt{1+8 n \bar{k}}}{2} & \text { if } n \geq 2 \bar{k}\end{cases}
$$

The approximations are shown by dotted line and the dot-solid line respectively in Figure 1. A derivation for the case when $n \gg \bar{k}$ can be found in Appendix C. 


\subsubsection{Quantity of resource $\langle S\rangle$ being allocated}

The expected quantity of resource $\langle S\rangle$ being allocated can thus be evaluated by using a similar argument. First, let us consider the event \{Exactly $w$ clients will be allocated with resources and $x$ quantity of resource will be allocated $\}$, i.e. $\{S(w, n) \leq x$ and $S(w+1, n) \geq \bar{k}\}$. Obviously, $\bar{k}-1 \leq x \leq \bar{k}$. Since $\{S(w, n) \leq x\}$ equals

$$
\{S(w, n) \leq x \text { and } S(w+1, n) \leq \bar{k}\} \bigcup\{S(w, n) \leq x \text { and } S(w+1, n) \geq \bar{k}\}
$$

and the first event is equivalent to $\{S(w+1, n) \leq \bar{k}\}$, it is readily shown that

$$
\begin{aligned}
& \operatorname{Pr}\{S(w, n) \leq x \text { and Exactly } w \text { clients being allocated }\} \\
= & \operatorname{Pr}\{S(w, n) \leq x \text { and } S(w+1, n) \geq \bar{k}\} \\
= & \operatorname{Pr}\{S(w, n) \leq x\}-\operatorname{Pr}\{S(w+1, n) \leq \bar{k}\}
\end{aligned}
$$

for all $x \in\{y \mid \operatorname{Pr}\{S(w, n) \leq y\}-\operatorname{Pr}\{S(w+1, n) \leq \bar{k}\} \geq 0\}$. Let

$$
h(x \mid w, n, \bar{k})=\operatorname{Pr}\{S(w, n)=x \mid \text { Exactly } w \text { clients being allocated }\} .
$$

It can thus be evaluated as follows :

$$
\begin{aligned}
& h(x \mid w, n, \bar{k})=\frac{d}{d x}\left\{\frac{\operatorname{Pr}\{S(w, n) \leq x\}-\operatorname{Pr}\{S(w+1, n) \leq \bar{k}\}}{\operatorname{Pr}\{S(w, n) \leq \bar{k}\}-\operatorname{Pr}\{S(w+1, n) \leq \bar{k}\}}\right\} \quad \text { if } w<n \\
& h(x \mid w, n, \bar{k})=\frac{d}{d x}\left\{\frac{\operatorname{Pr}\{S(n, n) \leq x\}}{\operatorname{Pr}\{S(n, n) \leq \bar{k}\}}\right\} \quad \text { if } w=n .
\end{aligned}
$$

for all $x \in\{y \mid \operatorname{Pr}\{S(w, n) \leq y\}-\operatorname{Pr}\{S(w+1, n) \leq \bar{k}\} \geq 0\}$. The expected quantity of resource being allocated, $\langle S\rangle$, can thus be written as follows :

$$
\langle S\rangle=\sum_{w=\bar{k}}^{n-1} \int_{x_{w}}^{\bar{k}} x d(\operatorname{Pr}\{S(w, n) \leq x\}-\operatorname{Pr}\{S(w+1, n) \leq \bar{k}\})+\int_{0}^{\bar{k}} x d \operatorname{Pr}\{S(n, n) \leq x\},
$$

for all $n \geq \bar{k}$ and $x_{w}$ satisfies the condition :

$$
\operatorname{Pr}\left\{S(w, n) \leq x_{w}\right\}=\operatorname{Pr}\{S(w+1, n) \leq \bar{k}\}
$$

Figure 2 shows the case when $\bar{k}$ equals to 20 . 
For large $n$, an approximated equation for the expected quantity of resource being allocated can be derived. Considering the residue, $R(n,\langle b\rangle, \bar{k})=\bar{k}-\sum_{i=1}^{\langle b\rangle} \frac{i}{n}$ satisfies the following inequality : $0 \leq R(n,\langle b\rangle, \bar{k}) \leq(\langle b\rangle+1) / n$ and supposing that this residue is uniform distributed on $[0,(\langle b\rangle+1) / n]$. The expected residue, $\langle R\rangle$ can be written as follows : $\langle R\rangle=(\langle b\rangle+1) / 2 n$. Substituting the approximation for $\langle b\rangle$ in Equation (11), the approximation of the expected quantity of resource being allocated can be written as follows :

$$
\langle S\rangle \approx \bar{k}\left(\frac{\sqrt{1+8 n \bar{k}}-3}{\sqrt{1+8 n \bar{k}}-1}\right)
$$

for $n \gg \bar{k}$. Reader can also refer to Appendix $\mathrm{C}$ for a derivation of the above equation.

\section{$3.2 \quad$ First-come-first-serve}

For the case that the resource is allocated in a first-come-first-serve basis, we consider the following equation : $S_{r}(w, n)=\sum_{i=1}^{w} k_{i}$, for all $\bar{k} \leq w \leq n$. By replacing $S(w, n)$ by $S_{r}(w, n)$, we can use the same argument used for greedy method to derive the equations for the expected number of clients being allocated with resource $\left\langle b_{r}\right\rangle$ and the expected quantity of resource $\left\langle S_{r}\right\rangle$ being allocated.

\subsubsection{Number of clients $\left\langle b_{r}\right\rangle$ being allocated with resource}

The expected number of clients being allocated with resources $\left\langle b_{r}\right\rangle$ can be determined by the following formula.

$$
\left\langle b_{r}\right\rangle=\sum_{w=\bar{k}}^{n-1} w\left\{\operatorname{Pr}\left\{S_{r}(w, n) \leq \bar{k}\right\}-\operatorname{Pr}\left\{S_{r}(w+1, n) \leq \bar{k}\right\}\right\}+n \operatorname{Pr}\left\{S_{r}(n, n) \leq \bar{k}\right\} .
$$

The experession for $\operatorname{Pr}\left\{S_{r}(w, n) \leq x\right\}$ will be from Feller formula [1].

$$
\operatorname{Pr}\left\{S_{r}(w, n) \leq x\right\}=\frac{1}{w !} \sum_{i=0}^{w}(-1)^{i} C_{i}^{w}(x-i)_{+}^{w},
$$

where

$$
x_{+}=\frac{x+|x|}{2}, \text { and } C_{i}^{w}=\frac{w !}{i !(w-i) !} .
$$


It should be noted that $\operatorname{Pr}\left\{S_{r}(w, n) \leq x\right\}$ is independent of $n$. Figure 3 shows the expected number of clients being allocated with resources against number of customers $n$ for the case that $\bar{k}=20$.

\subsubsection{Quantity of resource $\left\langle S_{r}\right\rangle$ being allocated}

Using the same technique as for $\langle S\rangle$, the expected number of units being sold $\left\langle S_{r}\right\rangle$ can be determined by the following equation.

$$
\left\langle S_{r}\right\rangle=\sum_{w=\bar{k}}^{n-1} \int_{x_{w}}^{\bar{k}} x d\left\{\operatorname{Pr}\left\{S_{r}(w, n) \leq \bar{k}\right\}-\operatorname{Pr}\left\{S_{r}(w+1, n) \leq \bar{k}\right\}\right\}+\int_{0}^{\bar{k}} x d \operatorname{Pr}\left\{S_{r}(n, n) \leq x\right\},
$$

for all $n \geq \bar{k} . x_{w}$ satisfies the condition :

$$
\operatorname{Pr}\left\{S_{r}(w, n) \leq x_{w}\right\}=\operatorname{Pr}\left\{S_{r}(w+1, n) \leq \bar{k}\right\}
$$

Figure 4 shows the case when $\bar{k}=20$. It should be noted that the expected quantity being allocated by FCFS method is slightly larger than the expected quantity being allocated by profit density based greedy algorithm, Figure 5.

\section{Greedy algorithm versus FCFS}

The results obtained in this section are summarized in Table 2. Without loss of generality, the results obtain in this paper can be extended for the case when $k_{i}$ is uniformly distributed on the range $[0, M]$ :

$$
\begin{array}{ll}
\text { Maximize } & \sum_{i=1}^{n} p_{i} s_{i} \\
\text { Subject to } & \sum_{i=1}^{n} k_{i} s_{i} \leq N \\
& s_{i} \in\{0,1\} \forall i=1, \ldots, n .
\end{array}
$$

Here $N$ is the total number of resource available.

By comparing the number of clients being allocated with resources, it is found that there is no difference between greedy algorithm or FCFS when the number of customers is less than $2 \bar{k}$. When $n>2 \bar{k}$, greedy algorithm can allocate resource to more clients than FCFS 


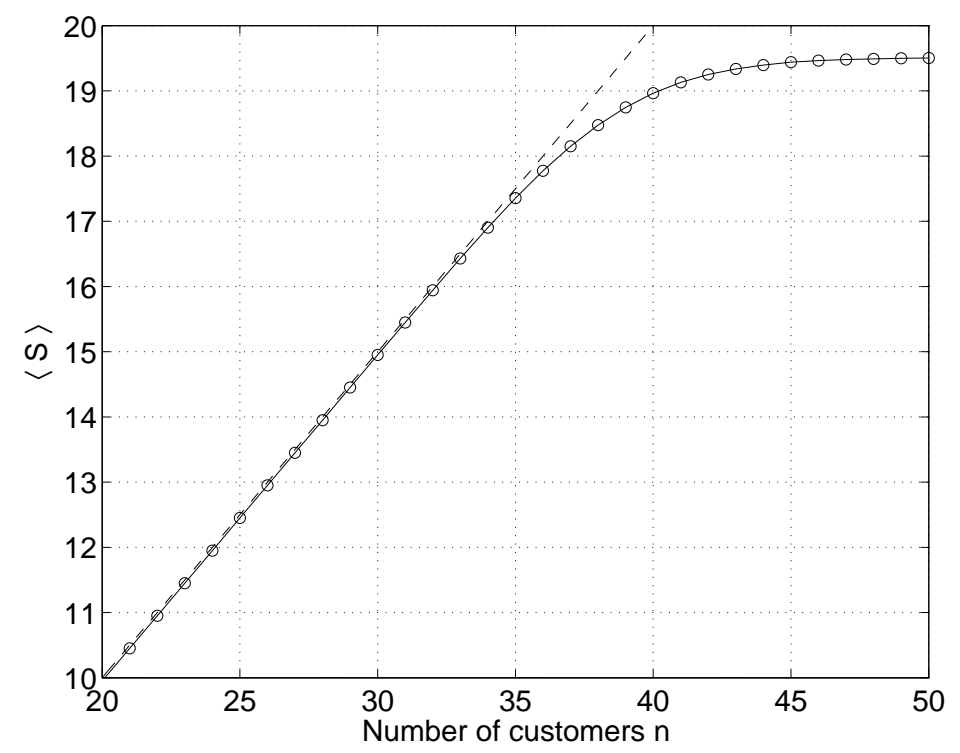

Figure 2: The expected quantity of resource being allocated $\langle S\rangle$ against the number of customers $n$ for $\bar{k}=20$.

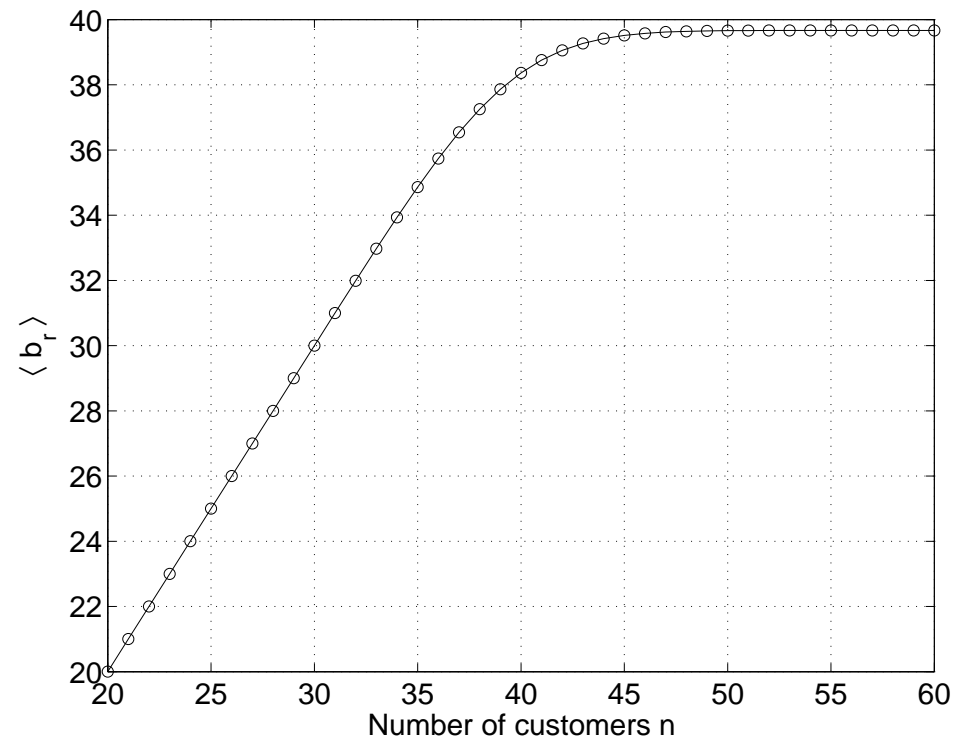

Figure 3: The expected number of clients being allocated $\left\langle b_{r}\right\rangle$ with resource against the number of customers $n$ for $\bar{k}=20$. 


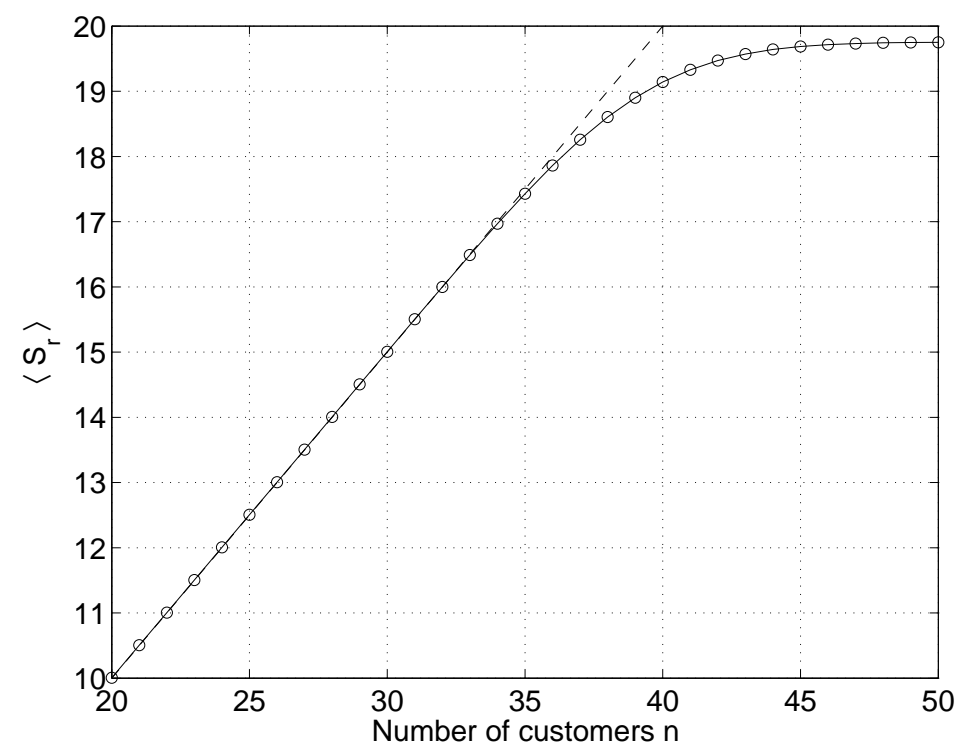

Figure 4: The expected quantity of resource being allocated $\left\langle S_{r}\right\rangle$ against the number of customers $n$ for $\bar{k}=20$.

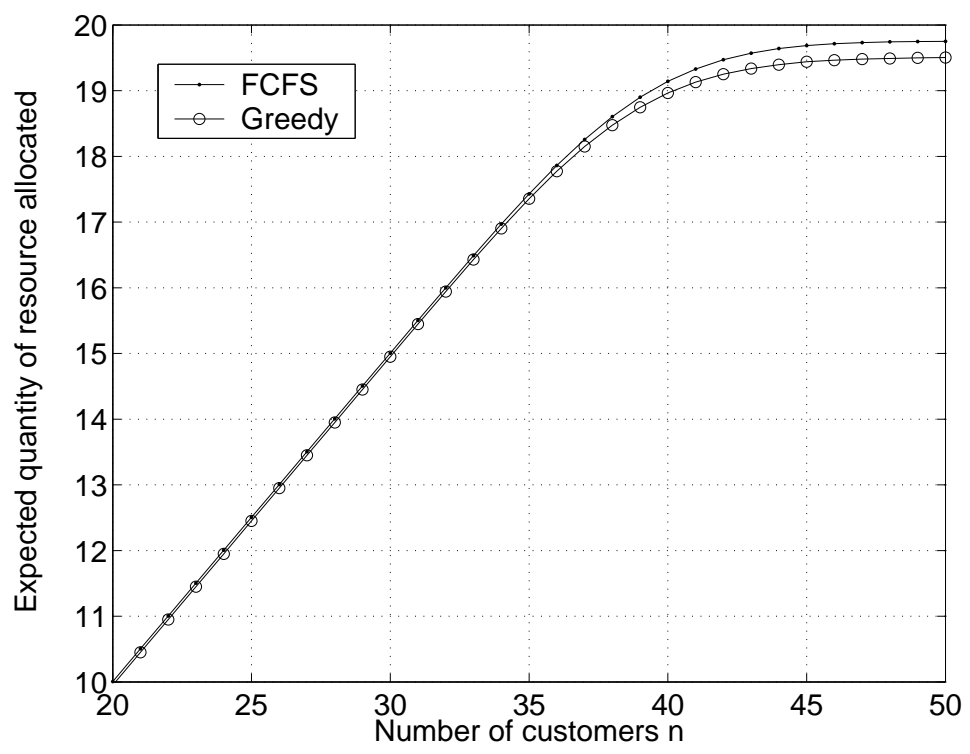

Figure 5: Comparison between FCFS and the greedy method in terms of the expected quantity of resource being allocated $\langle S\rangle$ (solid line with circles) and $\left\langle S_{r}\right\rangle$ (solid line with dots) for $\bar{k}=20$. 
method. Obviously, the resources are allocated to those clients whose requested quantities are comparatively small. On the other hand, by comparing the expected quantity of resources being allocated, FCFS can allocate more resources than greedy algorithm, Figure 5, irrespective to the number of customers $n$.

Suppose only the client who can get resources will have to pay and the service charge is defined as $P_{0}+P_{1} k_{i}, P_{0}, P_{1}>0$. The constant price $P_{0}$ can be interpreted as a premier that every client has to pay and $P_{1}$ can be interpreted as the unit resource price. The expected profit the WSP can gain by using greedy algorithm and FCFS respectively can be written as follows :

$$
\begin{aligned}
G & =P_{0}\langle b\rangle+P_{1}\langle S\rangle . \\
G_{r} & =P_{0}\left\langle b_{r}\right\rangle+P_{1}\left\langle S_{r}\right\rangle .
\end{aligned}
$$

With reference to the numerical results (Figure 5) obtained for $n=50$ (i.e. $n=2.5 \bar{k}$ ), it is clear that the difference between $\langle S\rangle$ and $\left\langle S_{r}\right\rangle$ is about $0.013 \times \bar{k}$ and the difference between $\langle b\rangle$ and $\left\langle b_{r}\right\rangle$ is about $0.2 \times \bar{k}$. Therefore, the difference between $G$ and $G_{r}$ can be expressed as follows :

$$
G-G_{r}=0.2 P_{0} \bar{k}-0.013 P_{1} \bar{k}
$$

Accordingly, $G>G_{r}$ if $P_{0} / P_{1}>0.013 / 0.2$. The profit gain by using greedy algorithm will be more than using FCFS. If $P_{0} / P_{1}<0.013 / 0.2$. profit gain by using FCFS will be more.

Of course, this comparison is only valid if the service charge model is linear. For other service charge models, a conclusion cannot easily be achieved. Numerical analysis will be needed.

\section{Conclusion}

In this paper, we have analyzed two properties of the profit density based greedy algorithm for a resource allocation problem in web service. The allocation problem is essentially a well know knapsack problem. In terms of profit making, greedy algorithm can ensure a near optimal solution. However, profit making is sometimes not the only consideration in management 
decision. Other properties such as the number of clients being allocated with resources and the quantity of resource being allocated are also important. In this regard, we have given a numerical analysis on these properties with respect to the profit density based greedy algorithm and the first-come-first-serve method.

The major tools that we used in the analysis are (i) the application of a formula derived by H. Weisberg in [6] for a linear combination of order statistics to analyze the greedy algorithm and (ii) the application of a formula derived by W. Feller in [1] for sum of uniform random variables to analyze the FCFS method. In accordance with the numerical results obtained, it is found that both profit density based greedy algorithm and FCFS method have very similar properties when the number of customers is not large, i.e. $n \leq 2 \bar{k}$. If $n=2.5 \bar{k}$, greedy algorithm has an advantage in letting more clients have resources allocated.

We have not concluded which algorithm is the best algorithm in resource allocation since resource allocation is itself a complicated problem, in particular when other management decisions are concerned. What we have presented here is simply additional remarks on profit density based greedy algorithm.

\section{A Weisberg formula - Linear combination of order sta- tistics}

To analyze the expected $\sum_{i=1}^{b} k_{i: n}$, we apply the formula derived by H.Weisberg [6] for linear combination of order statistics. Let

$$
S(n)=d_{1} U_{1: n}+\ldots+d_{n} U_{n: n},
$$

where $U_{i: n}$ is the $i^{\text {th }}$ order statistic drawn from uniform distribution $[0,1]$ and $d_{i}$ are real numbers. The probability for event $\{S(n) \leq x\}$ is given by the following formulae.

$$
\operatorname{Pr}\{S(n) \leq x\}=1-\sum_{j=1}^{r} \frac{\left(c_{j}-x\right)^{n}}{c_{j} \prod_{j \neq i}\left(c_{j}-c_{i}\right)}
$$

where $c_{i}$ 's are given as follows:

$$
c_{n+1}=0 ; \quad c_{k}=c_{k+1}+d_{k} .
$$


$\operatorname{Pr}\{S(n)<x\}$ is defined for all $0 \leq x \leq d_{1}+d_{2}+\cdots+d_{n}$ and $r$ is the largest integer such that $x \leq c_{r}$.

Illustrative example: Suppose $S(n)=U_{1: n}+U_{2: n}+U_{3: n} . d_{1}=d_{2}=d_{3}=1$ and $d_{i}=0$ for all $i=4, \ldots, n$. Then all the $c_{i}$ s will be given as follows :

$$
c_{1}=3 \quad c_{2}=2 \quad c_{3}=1 \quad c_{4}=0 \quad \ldots \quad c_{n+1}=0 .
$$

The cdf can be written as following equations.

$$
\begin{array}{ll}
\forall x \geq 3 & \operatorname{Pr}\{S(n) \leq x\}=1 \\
\forall 2 \leq x<3 & \operatorname{Pr}\{S(n) \leq x\}=1-\frac{(3-x)^{n}}{3 \prod_{j \neq i}\left(3-c_{i}\right)} \\
\forall 1 \leq x<2 & \operatorname{Pr}\{S(n) \leq x\}=1-\frac{(3-x)^{n}}{3 \prod_{j \neq i}\left(3-c_{i}\right)}-\frac{(2-x)^{n}}{2 \prod_{j \neq i}\left(2-c_{i}\right)} \\
\forall 0 \leq x<1 & \operatorname{Pr}\{S(n) \leq x\}=1-\frac{(3-x)^{n}}{3 \prod_{j \neq i}\left(3-c_{i}\right)}-\frac{(2-x)^{n}}{2 \prod_{j \neq i}\left(2-c_{i}\right)}-\frac{(1-x)^{n}}{1 \prod_{j \neq i}\left(1-c_{i}\right)} \\
\forall x<0 & \operatorname{Pr}\{S(n) \leq x\}=0
\end{array}
$$

\section{B Feller formula - Sum of $n$ uniform random variables}

To analyze the expected $L$ of the case that the products are sold in first-come-first-serve basis, we need the following formulae derived by W. Feller (p.27 of [1]). Let $S_{r}(n)$ be the sum of uniform random variables defined as follows :

$$
S_{r}(n)=U_{1}+U_{2}+\ldots+U_{n}
$$

Noted that $U_{i}$ 's are not ordered. For $n=1,2, \ldots$ and $0 \leq x \leq n$,

$$
\operatorname{Pr}\left\{S_{r}(n) \leq x\right\}=\frac{1}{n !} \sum_{v=0}^{n}(-1)^{v} C_{v}^{n}(x-v)_{+}^{n},
$$

where

$$
x_{+}=\frac{x+|x|}{2} \text {, and } C_{v}^{n}=\frac{n !}{v !(n-v) !} .
$$

Note that for a point $x$ between $(k-1)$ and $k$ only $k$ terms of the sum are different from zero. 
Illustrative example: Let $S_{r}(3)=U_{1}+U_{2}+U_{3}$ and $U_{i}$ s are not in order, the cdf can be written as following equations.

$$
\begin{array}{ll}
\forall x \geq 3 & \operatorname{Pr}\left\{S_{r}(3) \leq x\right\}=1 \\
\forall 2 \leq x<3 & \operatorname{Pr}\left\{S_{r}(3) \leq x\right\}=\frac{C_{0}^{3} x^{3}-C_{1}^{3}(x-1)^{3}+C_{2}^{3}(x-2)^{3}}{3 !} \\
\forall 1 \leq x<2 & \operatorname{Pr}\left\{S_{r}(3) \leq x\right\}=\frac{C_{0}^{3} x^{3}-C_{1}^{3}(x-1)^{3}}{3 !} \\
\forall 0 \leq x<1 & \operatorname{Pr}\left\{S_{r}(3) \leq x\right\}=\frac{C_{0}^{3} x^{3}}{3 !} \\
\forall x<0 & \operatorname{Pr}\left\{S_{r}(3) \leq x\right\}=0
\end{array}
$$

\section{Derivation of Equation (11) \& Equation (16)}

For $n$ is large and $n \gg \bar{k}$, we assume that the $k_{i}$ s are distributed evenly on $[0,1]$. Without loss of generality, we futher assume $k_{i}<k_{j}$ if $i<j$. So, the values of $k_{i}$ s can be written as

$$
k_{i}=\frac{i}{n}
$$

for all $i=1,2, \cdots, n$. As greedy allocation implies, the resource will be allocated to $k_{1}, k_{2}$ and so on until futher allocation is not possible. Let $\langle r\rangle$ be the last one who can be allocated with resource. It turns out that

$$
\sum_{i=1}^{\langle b\rangle} k_{i} \approx \bar{k}
$$

It is equivalent to that

$$
\begin{aligned}
\sum_{i=1}^{\langle b\rangle} \frac{i}{n} & \approx \bar{k} \\
\frac{\langle b\rangle^{2}+\langle b\rangle}{2 n} & \approx \bar{k} .
\end{aligned}
$$

The solution of $\langle b\rangle$ is thus approximately equal to $\frac{-1+\sqrt{1+8 n \bar{k}}}{2}$. Since $\langle R\rangle=(\langle b\rangle+1) / 2 n$,

$$
\begin{aligned}
\langle S\rangle & \approx \bar{k}-\frac{\langle b\rangle+1}{2 n} \\
& =\bar{k}-\frac{1+\sqrt{1+8 n \bar{k}}}{4 n} \\
& =\bar{k}\left(1-\frac{1+\sqrt{1+8 n \bar{k}}}{4 n \bar{k}}\right)
\end{aligned}
$$




$$
\begin{aligned}
& =\bar{k}\left(1-\frac{8 n \bar{k}}{4 n \bar{k}(\sqrt{1+8 n \bar{k}}-1)}\right) \\
& =\bar{k}\left(\frac{\sqrt{1+8 n \bar{k}}-3}{\sqrt{1+8 n \bar{k}}-1}\right) .
\end{aligned}
$$

Whenever $n$ is large, $\langle S\rangle \rightarrow \bar{k}$.

\section{Acknowledgement}

The authors would like to thank for the reviewers giving valuable comments on the earlier version of this paper.

\section{References}

[1] W. Feller, Probability Theory: Volume II, Wiley, 1971.

[2] B. Lehmann, D. Lehmann and N. Nisan, Combinatorial auctions with decreasing marginal utilities, EC'01, Tampa, Florida, 2001.

[3] E.S. Maskin and J.G. Riley, Optimal multi-unit auctions, The Economics of Missing Markets, Information, and Games, Oxford University Press, 1989.

[4] S. Martello and P. Toth, Knapsack Problems: Algorithms and Computer Implementations, John Wiley \& Sons, 1990.

[5] C.H. Papadamitrious and K. Steiglitz, Combinatorial Optimization: Algorithms and complexity, Dover, 1998.

[6] H. Weisberg, The distribution of linear combinations of order statistics from the uniform distribution. Annals of Mathematical Statistics, Vol.42, 704-709, 1971. 


\begin{tabular}{l|ll}
\hline (a) & Greedy Algorithm & FCFS \\
\hline$n \leq 2 \bar{k}$ & $\langle b\rangle \approx n$ & $\left\langle b_{r}\right\rangle \approx n$ \\
& $\langle S\rangle \approx n / 2$ & $\left\langle S_{r}\right\rangle \approx n / 2$ \\
$n \gg 2 \bar{k}$ & $\langle b\rangle=(-1+\sqrt{1+8 n \bar{k}}) / 2$ & $\left\langle b_{r}\right\rangle \approx \bar{k}$ \\
& $\langle S\rangle \approx \bar{k}$ & $\left\langle S_{r}\right\rangle \approx \bar{k}$ \\
\hline (b) & Greedy Algorithm & FCFS \\
\hline$n \leq 2 N / M$ & $\langle b\rangle \approx n$ & $\left\langle b_{r}\right\rangle \approx n$ \\
& $\langle S\rangle \approx n / 2$ & $\left\langle S_{r}\right\rangle \approx n / 2$ \\
$n \gg 2 N / M$ & $\langle b\rangle=(-1+\sqrt{1+8 n N / M}) / 2$ & $\left\langle b_{r}\right\rangle \approx N / M$ \\
& $\langle S\rangle \approx N$ & $\left\langle S_{r}\right\rangle \approx N$ \\
\hline
\end{tabular}

Table 2: (a) Summary on the expected number of customers and the expected number of units being sold for both auction and FCFS. (b) $N$ and $M(>1)$ correspond to the total number of units for sale and the $\max \left\{k_{i}\right\}$. 